\title{
Ueber die Erhaltung der Kraft.
}

\author{
Eine physikalische Abhandlung \\ von \\ Hermann von Helmholtz.
}

Vorbemerkung. Seite 60

\section{Einleitung.}

Gründe zur Erörterung der Einleitung und Beschränkung der ersteren. Wege zur Begrïndung der Erhaltung der Energie und letztes Ziel der Naturwissenschaft nach von Helmholtz. - Letzte Ursachen. Materie: »Materie an sich«. Masse. Chemische Elemente. - Spätere Auffassung der Kausalität bei von Helmholtz. - Bewegung. Punktkräfte. - Fernkräfte als letzte Ursachen. - Rückblick . Seite 6I

\section{Das Prinzip von der Erhaltung der lebendigen Kraft.}

Der Satz von Carnot-Clapeyron. - Der Satz von der Erhaltung der lebendigen Kraft. - Aufzählung der Hypothesen von von Helmholtz

Seite 93

Das Prinzip von der Erhaltung der Kraft.

Definition des Integrals nach von Helmholtz. - Seine Berechnung der Arbeit . . . . . . . . . . . . . . Seite 98 


\section{Die Anwendung des Prinzips in den mechanischen Theoremen.}

Aufzählung von Vorgängen, worin die Erhaltung der Energie bereits bekannt war. - Allgemeine Mutmassungen . . . . . . . Seite ror

\section{Kraftäquivalent der Wärme.}

Stoss und Reibung. - Wärmeäquivalent der Arbeit. - Arbeitsäquivalent der Wärme. - Wärme als Bewegung aufgefasst. - Chemische Wärme. - Erzeugung und Verschwinden von Wärme. - Was von Helmholtz als Herleitung des mechanischen Wärmeäquivalents bezeichnet. - Formeln von Clapeyron und Holtzmann. - Rückblick . . . Seite 102

\section{Kraftäquivalent der elektrischen Vorgänge.}

Gewinn an lebendiger Kraft bei der wechselseitigen Neutralisation zweier geladener Leiter. - Anwendung auf die Entladung der Leydener Flasche. - Elektrische Fluida. - Riickblick . . . . Seite I12

\section{Kraftäquivalent des Magnetismus und Elektromagnetismus.}

Magnetisierung eines Elsenstückes durch einen unveränderlichen Stahlmagnet. - Elektromagnetische Potentialbestimmungen. - Rückblick Seite 121

\section{Galvanismus.}

Kontaktkraft. - Konstante Ketten. - Polarisation. - Thermoströme. Rückblick . . . . . . . . . . . . . . . . . Seite I24

Anmerkung über die analytische Darstellung der Energie

\section{Das Verhalten von Hermann von Helmholtz gegen Robert Mayer

